\title{
Experimental Study on Hardening of High Speed Cold Roll-beating Formation
}

\author{
Hongyu $\mathrm{Xu}^{\mathrm{a}}$, Yanyi Huang ${ }^{\mathrm{b}}$, Yuhui Liu, Fengkui Cui \\ School of Mechatronics Engineering, Henan University of Science and Technology, Luoyang \\ 471003, China \\ aemail:xuhongyu@haust.edu.cn, bemail:115993902@qq.com,
}

Keywords: involute spline, cold roll-beating, work hardening, microhardness, microstructure

\begin{abstract}
In order to improve the surface quality of the cold roll-beating workpiece and explain the surface hardening behavior of the workpiece, an experiment on the microhardness and scanning electron microscopy of a cold roll-beating workpiece was carried out by using involute spline as the subject. The changes of the hardness of the splines at different positions under the same process parameters were analyzed, and the changes of the surface hardening degree and the microstructure of the splines under different process parameters were discussed. The results show that the surface hardness of the tooth top, index circle and tooth root under different process parameters decreases with the increase of the distance from the tooth surface. The degree of hardening of the spline surface, at the same rotate speed, increases as the feed rate increases and at the same feed rate, it decreases as the rotate speed increases.
\end{abstract}

\section{Introduction}

As an advanced plastic forming technology, cold roll-beating is widely used in spline, lead screw and gear processing. cold roll-beating principle, the law of metal flow in the process of cold roll-beating and cold roll-beating stress and strain were studied by the scholars, but the surface microstructure of cold roll-beating has little research involved. Work hardening can improve the strength, fatigue resistance, impact resistance, etc. of the workpiece surface, but excessive work hardening will make the microcracks and other defects. In order to control the degree of work hardening and improve the surface integrity of the cold roll-beating forming, the research on work hardening behavior of spline surface has important engineering application value.

$\mathrm{Lu}$ Zhang et al.[1] obtained the rule of metal flowing during the cold roll-beating process according to numerical simulation results of stress field. In summary, the research of cold roll-beating technology is mainly in the aspects of dynamics, kinematics, design and manufacture of roller, metal flow, formation mechanism of thermo-mechanical coupling and so on, and there are few reports about the work hardening behavior. Yaofeng LV et al.[2] respectively motion model of continuous indexing cold roll-beating spline was established to reveal the method for involute spline of cold roll-beating formation is generative. Wenjuan Zhu et al.[3] done the theoretical modeling analysis for multi field coupling mechanism during process of cold roll-beating based on the theory of dissipation. Zella L.et al.[4] analyzed the contact of symmetrical loading and non axis symmetric loading of the involute spline by finite element method, stress concentration distribution of two kinds of models was obtained. Qun Ma et al.[5] designed a improved rolling head structure and established a mathematical model to calculate the curve in the cross section of the key way formed by roll-beating wheel. Yuxi Li et al.[6]used Deform simulation software analyzed high speed cold roll-beating formation with different deformation zone, different temperature and different strain rate and the changes of stress, strain, temperature and metal flow during the process of high speed cold roll-beating formation were revealed, lay the foundation for further research on high speed cold roll-beating. Work hardening is an important evaluating index for the surface quality, which influenced by different processing parameters. Therefore, it is of great theoretical significance and engineering application value to study the influence of cold roll-beating parameters on the surface work hardening. 
The experiment of micro hardness and scanning electron microscope of spline under different machining parameters are carried out to study on work hardening behavior and the change of microstructure, and to analyze the change tendency of microhardness with the layer depth of cold roll-beating spline surface and the influence of machining parameters on the hardening and microstructure.

\section{Experimental scheme}

\section{Equipment.}

The processing of cold roll-beating involute spline is carried out on the ZRme9 roll-beating machine of Swiss Grob Company as shown in Fig.1. The surface hardness of the sample was measured by HVS-1000A micro hardness tester. Microstructure of spline tooth specimen was observed under JSM-5610LV scanning electron microscope (SEM) as shown in Fig.2.

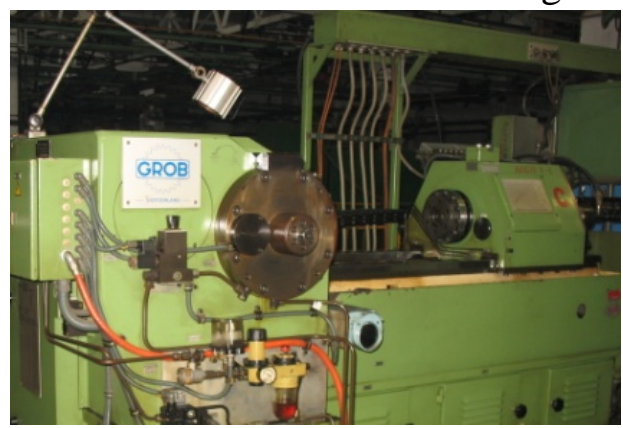

Fig.1. ZRMe9 roll-beating machine

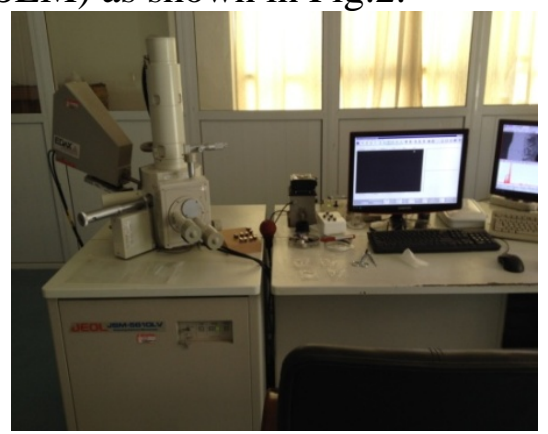

Fig.2. HVS-1000A micro hardness tester

Materials and parameters.

Blank material of involute spline is 1020 steel, chemical composition of 1020 steel as shown in Table 1. The modulus of involute spline is 2.5 , the number of teeth is 14 , the pressure angle is $30^{\circ}$, addendum coefficient ha ${ }^{*}=0.5$ and dedendum coefficient $\mathrm{hf}^{*}=0.75$, Experimental rotation speed of roll-beating wheel is 1428, 1581, 1806, 2032 and $2258 \mathrm{r} \bullet \mathrm{min}^{-1}$, feed rate of the workpiece is 21, 28, 35 and $42 \mathrm{~mm} \cdot \mathrm{min}^{-1}$.

Table 1 Chemical composition of 1020 steel (mass fraction, \%)

\begin{tabular}{ccccccc}
\hline $\mathrm{C}$ & $\mathrm{Si}$ & $\mathrm{Mn}$ & $\mathrm{P}$ & $\mathrm{S}$ & $\mathrm{Ni}$ & $\mathrm{Cr}$ \\
\hline \multirow{2}{*}{0.20} & $0.17 \sim 0.3$ & $0.35 \sim 0.6$ & $\leq 0.035$ & $\leq 0.035$ & $\leq 0.30$ & $\leq 0.25$ \\
\hline
\end{tabular}

\section{Procedure.}

The process of cold roll-beating involute spline on the rolling machine is adopted in pulling out beat. The spline is processed as shown in Fig.3. Cut a tooth using a wire cutting machine. A section is cut along the tooth as a sample as shown in Fig.4, and then grinds and polishes it.

The sample hardness are measured by micro hardness tester along the surface normal from the surface to the inside, vivtorinox hardness of each section measures 20 points, the distance between each measuring point that load $100 \mathrm{~g}$ and $10 \mathrm{~s}$ is $0.1 \mathrm{~mm}$.

After cleaning the surface by the alcohol cotton, the cross-section of samples are treated 50s by nital with concentration of $4 \%$ until present a light brown, dry after cleaning. Observe the surface microstructure of addendum, pitch and dedendum of spline samples under the scanning electron microscope, the electron acceleration voltage is $20 \mathrm{kV}[7][8]$. 


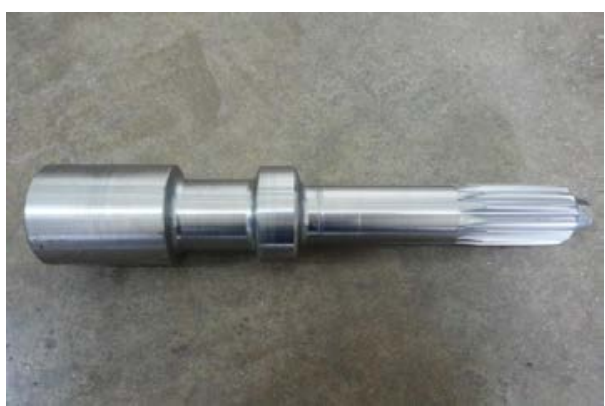

Fig.3. Spline of Cold roll-beating

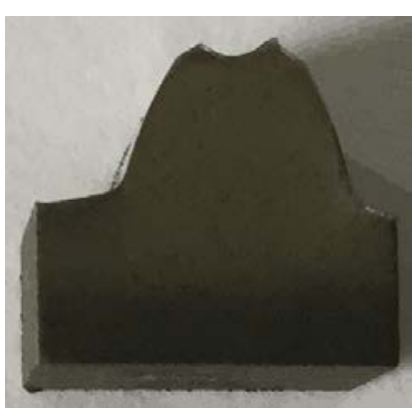

Fig.4. Spline sample

\section{Result.}

\section{1)Result and analysis of micro hardness experiment under the same parameters.}

At the same processing parameters, the hardness curve of addendum, pitch and dedendum of spline along the normal direction of as shown in Fig.5, the figure shows the distribution of surface hardness, experimental rotation speed of roll-beating wheel is $2258 \mathrm{r} \bullet \mathrm{min}^{-1}$ and feed rate of the workpiece is $21 \mathrm{~mm} \cdot \mathrm{min}^{-1}$. It can be seen from the figure that the change tendency of hardness of each position is basically the same under different process parameters, which increases with the increase of the distance from tooth surface. The hardness of the three position is obviously improved compared with the matrix that hardness is $238 \mathrm{HV}$ forming an obvious work hardening layer.The maximum hardness of the addendum reaches $296.7 \mathrm{HV}$, the hardness decreases obviously with the increase of the normal distance along the surface, and then gradually tends to be stable, the thickness of the hardened layer is close to $0.5 \mathrm{~mm}$; The maximum hardness of the pitch is $316.7 \mathrm{HV}$, with the depth of the depth of measurement, Its hardness began to decline significantly ,a small piece of the middle is relatively flat, and then continue to decline, finally stabilized, depth of hardening layer is more than $1.20 \mathrm{~mm}$; The maximum hardness of dedendum is $339.4 \mathrm{HV}$ and hardness has been declining from surface to the depth direction and finally close to matrix hardness, the hardening layer to $1.5 \mathrm{~mm}$.

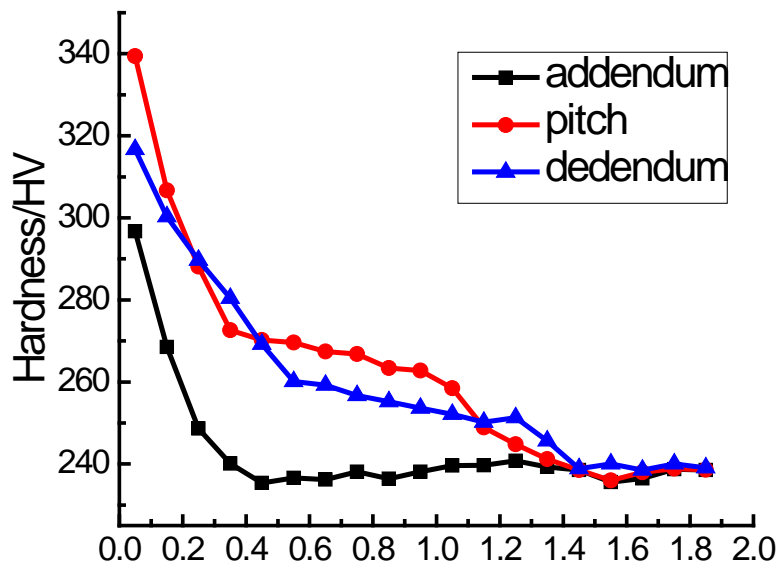

Normal distance from the surface/mm

Fig.5. Hardness distribution curve along the normal direction of addendum, pitch and dedendum under different process parameters

In the forming process, the first contacting with the roll-beating wheel forms into cogging where is the region of addendum. The whole tooth profile will be in contact with the roll-beating wheel with the increase of the impact depth, which is subjected to the constraint of the deformation direction and the combined effects of various stresses. Although the first region contacting with the roll-beating wheel is the region of addendum, the metal flow at the top of the tooth is relatively free and the extrusion force is not strong. As a result, the surface hardness of addendum is small and the hardening layer is relatively shallow. The pitch directly affected by the force when contacting with the roll-beating wheel, where plastic deformation is relatively large and surface hardness value is also relatively large, hardened layer is also deeper. Although the deformation degree of dedendum 
is the largest, the temperature rise softening effect is more obvious. So the surface hardness of dedendum is slightly lower than the surface hardness of the pitch.

2)Results and analysis of micro hardness experiment under different process parameters.

The degree of work hardening is an important index to evaluate the work hardening, which is indicated by the ratio of the micro hardness of the surface layer processing before and after. Curve of hardening degree of pitch under different feed rate as shown in Fig.6(a). From the figure, it can be seen that the hardening degree rises with the increase of the feed rate. The deformation of the workpiece enlarges with the increase of the feed rate, since the stress hardening effect of 1020 steel is obvious when the deformation is small and the rate of work hardening is higher. With the increase of deformation, the amount of heat produced by plastic deformation will increase and temperature rise softening effect will be obvious, But the change of deformation in a small range,stress strengthening effect is still dominant. Therefore, the hardening degree of spline gear increases with the increase of the feed rate.

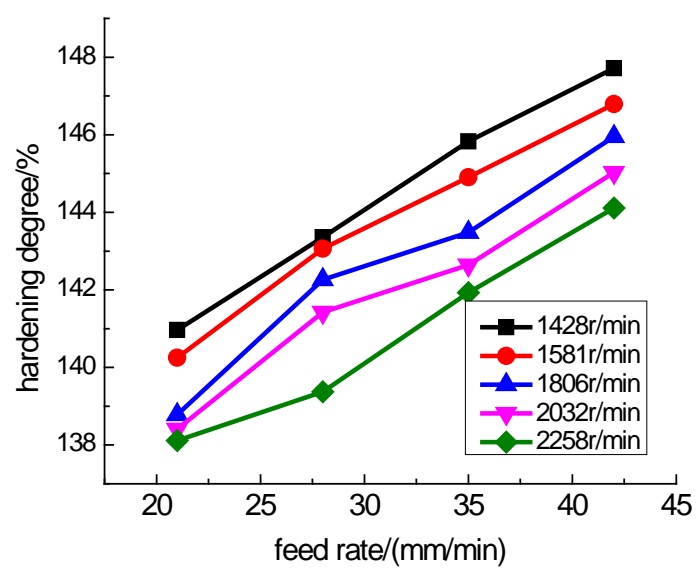

(a)

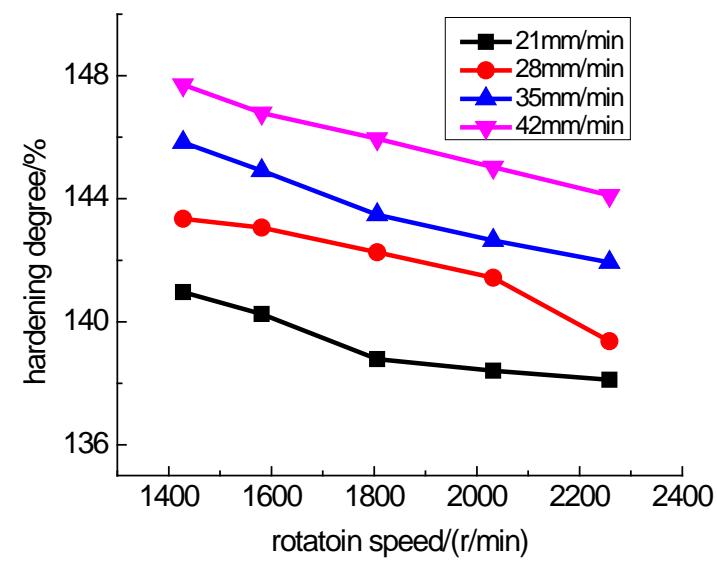

(b)

Fig.6. Hardening degree of graduated circle under different process parameters

Hardening degree curve of pitch under different rotation speed as shown in Fig.6(b), It can be seen that the hardening degree slightly decreased with the increase of the rotation speed. Although the rise of rotating peed will make the strain rate increase, dislocation motion be exacerbated, and play a role in the strengthening of stress, But the rise of rotating speed of the roll-beating wheel will increase the number of hits, The contact time is shortened, the heat produced by plastic deformation can not diffuse to the surrounding to increase the temperature of the workpiece, temperature rise softening effect will be obvious. The strain hardening phenomenon caused by the increase of the strain rate with the improvement of the rotation speed of the roll-beating wheel affected by surface thermal softening effect is weakened. Therefore, the degree of hardening decreases slightly with the increase of rotation speed.

3)Results and analysis of scanning electron microscope experiment.

The surface microstructures of spline tooth section under different process parameters are observed under 1000 times of electron microscope respectively as shown in Fig.7. The microstructure of each group from left to right is shown rotation speed $1428 \mathrm{r} \bullet \mathrm{min}-1$, feed rate $21 \mathrm{~mm} \bullet \mathrm{min}-1$; rotation speed $1428 \mathrm{r} \bullet \mathrm{min}-1$,feed rate $42 \mathrm{~mm} \bullet \mathrm{min}-1$; rotation speed $2258 \mathrm{r} \bullet \mathrm{min}-1$, feed rate $21 \mathrm{~mm} \bullet \mathrm{min}-1$. 

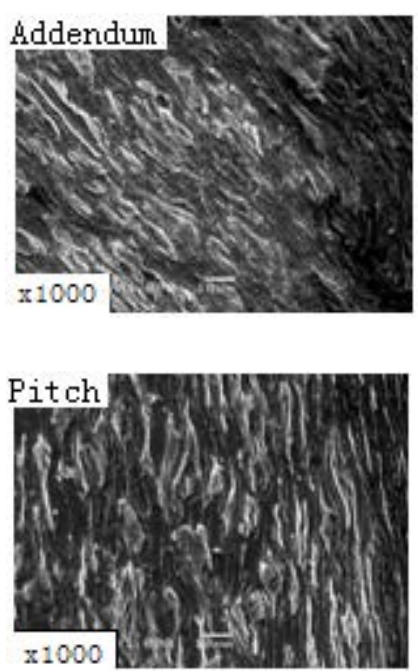

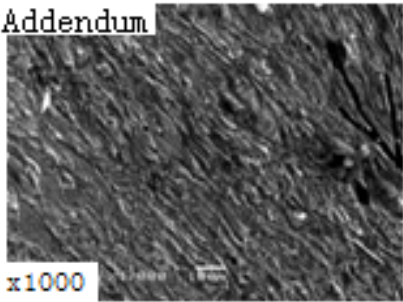

(a) addendum

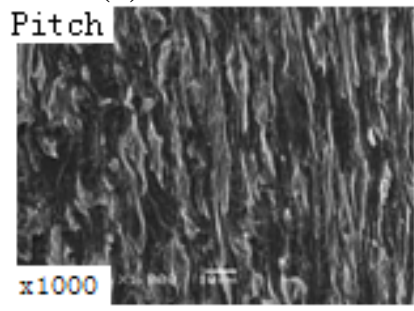

(b) pitch
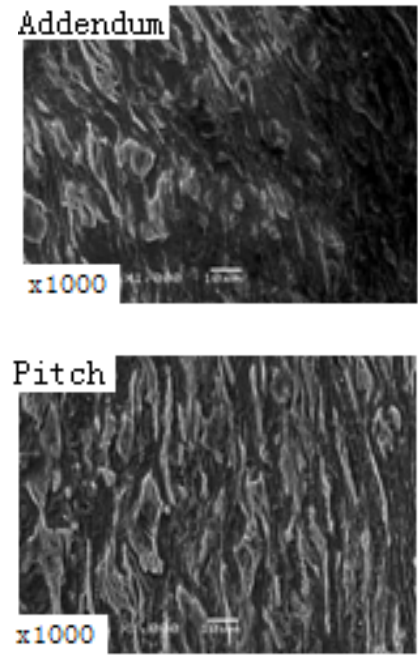

Fig.7. Microstructure of spline addendum and pitch under different process parameters

It is can be seen that the grains of spline tooth section changes in different position have obvious difference under the same processing parameters, the grain sizes in the position of dedendum vary the most drastically, and the grain is significantly elongated, which in the addendum stretching slightly and deformation degree is similar, deformation degree of pitch grain between dedendum and addendum. The metal of the roll-beating wheel flows to the blank area when the wheels hit the workpiece under the action of the impact of the roll-beating wheel on the workpiece[9]. With the increase of the deformation, the grains of the metal surface and its inclusion are crushed gradually, refined, and rotation, finally forms the bar fibrous tissue[10]. In the process of plastic deformation, compressive stress of addendum, pitch is different, which lead to the degree of grain refinement and the density of the fibrous tissue are different. The addendum is subjected to the pressure of the roll-beating wheel is relatively small, and metal flow resistance is smallest. Therefore, the change of metal microstructure is small. Because of roll-beating wheel act on both sides of the spline tooth, the pressure on both sides of cogging is bigger than addendum, so the fiber tissue density at the pitch is larger than the addendum. The deformation degree of crystals of the tooth surface is also different under different processing parameters. The greater the feed rate, the smaller the spindle speed, the more dense the fibrous tissue[11][12].

The degree of deformation of the metal and the density of the fiber had a direct influence on the degree of work hardening.From the changes by the organization of the metal we can see that the hardness value of addendum is the smallest, which of pitch is bigger, and the degree of work hardening is increased with the increase of the feed rate, and decreases with the increase of the rotation speed. The result is consistent with the microhardness experiment results.

\section{Conclusion}

1). The change trends of surface hardness in different locations are basically the same under the same process parameters, which tends to decrease with the increase of the tooth surface distance. Matrix hardness of the workpiece is $238.5 \mathrm{HV}$, the hardness of addendum, pitch and dedendum are significantly improved forming obvious work hardening layer,harden layer to $1.5 \mathrm{~mm}$

2). Under different processing parameters, the degree of work hardening is different. which increases with the increase of feed rate at the same rotation speed, and decreases with the increase of rotation speed under the same feed rate.

3). During the process of plastic formation, the grains of the metal surface and the inclusions are gradually crushed, refined, then eventually form a strip of fibrous tissue. Addendum and pitch get different pressure stress, and where deformation is also different, resulting the degree of grain refinement and the density of fibrous tissue is different,The hardness value will be higher where deformation is large and fibrous tissue is dense. 


\section{References}

[1] Zhang L, Li Y, Yang M S, et al. Study on Metal Flowing of Lead Screw Cold Roll-beating Forming[J]. China Mechanical Engineering, 2012, 23(13):1623-1628.

[2] Lu Y F, Cui F K, Yang J X, et al. The Forming Theory and Error Calculating of Cold Rolling Involute Spline[J]. Combination Machine Tools and Automatic Processing Technology, 2007, 34 (5):20-23.

[3] Cui F K, Zhu W J, Liu J G, et al. Thermo mechanical coupling simulation of high speed cold roll-beating[J]. Journal of Henan University of Science and Technology, 2014, 35(1):13-16.

[4]Zella L., Kahn-Jetter, Suzanne Wright. Finite Element Analysis of an Involute Spline[J]. Journal of Mechanical Design, 2000, 122(2):239-244.

[5] Ma Q, Li Y, Yang M S, et al. Structure Improvement of Roll-beating Head for Continuous Indexing Cold Roll-beating Machine[J]. Acta Armamentarii, 2015, 36(8):1587-1593.

[6] Li Y X, Li Y, Yang M S, et al. Investigation of The Tooth Profile Error Based on the Forming Theory of Balloscrew Manufactured by Cold Rolling[J]. Act a Armament, 2015, 36(8): 1594-1600.

[7] Zhang W, Zheng $\mathrm{M} \mathrm{L}, \mathrm{Xu} \mathrm{J} \mathrm{H}$, et al. Surface work hardening experiment of titanium alloy Ti-6Al-4V Turning[J]. Journal of Harbin Engineering University, 2013, 34(8):1052-1056.

[8] Zhou Z T, Chen Z T, Jiang L K, et al. Experiment on work hardening of milling TB6[J]. Journal of Beihang University, 2014, 40(1):135-140.

[9] Cui F K, Xie Y F, Dong X D, et al. Simulation analysis of metal flow in high-speed cold roll-beating[J]. Journal of Henan Polytechnic University (Natural Science), 2014, 33(4):467-471.

[10] Wang X Q, Cui F K, Yan G P, et al. Study on Dislocation Density Change during Cold Roll-beating of 40Cr[J]. China Mechanical Engineering, 2013, 24(16):2248-2256.

[11] Cui F K. Research on High Speed Precision Cold Rolling Forming Technology[D]. Xi'an: Xi'an University of Technology, 2007.

[12] Sum Hongyum, Li Jun, Meng Qingge, et al. Effect of heating rates on microstructure and bake hardening behavior of DP steel[J]. Forging \& Stamping Technology, 2014, 4(39):115-121. 